\title{
Instantaneous detection of neuro-oscillators using a portable tool
}

\author{
F. Brunetti ${ }^{1}$, E. Rocon ${ }^{1}$, M. Manto ${ }^{2}$ and J.L. Pons ${ }^{1}$ \\ ${ }^{1}$ Instituto de Automática Industrial, CSIC (Spain) \\ ${ }^{2}$ Université Libre de Bruxelles, ULB (Belgium) \\ Email: brunetti@iai.csic.es
}

\begin{abstract}
The TCA (Tremor Coherence Analyzer) project proposes a portable system to asses instantaneously muscle coupling using surface electromyography (SEMG). The present article shows preliminary results that justify the use of this portable tool to detect sudden emergence of neuro-oscillators during typical diagnosis tasks in people with pathological tremor. The final aim of the presented system is to assess oscillators coupling instantaneously. This type of tool is very helpful in order to study neuromotor disorders such as tremor and to assess drug therapy.
\end{abstract}

\section{INTRODUCTION}

The use of the coherence to estimate coupling between signals is very common. EEG and EMG signals have been mainly used to study neuromotor patterns [1][2]. The coherence function is a spectral method that allow us to estimate the coupling of signals by basically comparing their spectral composition in a normalized way. The use of this method has many applications in the field of neurophysiology [3][4]. Some of these applications are:

1) Parkinson's disease (PD) tremor exhibits a high intralimb but lower interlimb coupling between SEMG in flexor and extensor muscles. In PD, a mental task increases the coherence between muscles of the same limb, whereas a finger-to-nose test decreases the coherence [5].

2) When both cranial and limb muscles show high coherence values, a supra-spinal mechanism can be suspected.

3) Compensatory mechanisms during movement can be estimated.

4) Effects of drugs on the oscillatory behavior of the nervous and musculoskeletal systems can be investigated.

5) Analysis of episodes of bilateral high-frequency synchronous discharges can lead to a better understanding of tremor genesis [6].

In these instances, coherence values higher than 0.6 are usually considered as high. In some disorders, values between 0.9 and 1 are reached.

The TCA project, [7], developed a portable system to assess coupling between muscles based on SEMG signals. Once the system has been developed, during first trials, the study of the sudden activation of neuro-oscillators during specific tasks and the relation with possible compensatory strategy becomes a new purpose of the system. Because of this, the TCA system represents a step forward in the detection of oscillators as a way to study neuromotor disorders such as tremor. The aim of the article is to show the applications of this new type of tools.

First we present briefly the features of the system. The second part of the paper shows the analytical methods employed to process the SEMG signal. The last part of the article evaluates these mentioned applications based on the preliminary results of the TCA system with a set patients with pathological tremor.

\section{SEMG DATA ANALYSIS}

A large number of techniques, both in time and the frequency domain, can be used to analyze data from SEMG sensors. One of them is the coherence function, that is generally used to estimate the linearity between two signals. The coherence function utilizes the the spectral estimation of two signals to compare them and establish a measure of the linearity. Because, in general, we deal with random signals, the use of an estimation of the spectrum is required. The mean value calculation reduces the randomness of a signal and makes smoother the signal spectra. But the simple mean value of the Fourier transform is not enough since averaging random complex numbers would vanish.

\section{A. Spectral estimation}

The aim of the power spectrum is to provide a distribution of the energy over the spectrum of a signal $x[n]$ and it is defined by

$$
P_{x x}(f)=\sum_{-\infty}^{+\infty} R_{x x}(m) e^{j 2 \pi f m} .
$$

where $R_{x x}(m)$ is the autocorrelation function.

To avoid the effect of randomness in spectral calculation, we use the mean square value of the Fourier transform, usually referred to as the periodogram. Thus, when we have a discrete random signal $x[n]$ of $N \cdot L$ length points, called epoch, we will divide this signal into $N$ segments of $L$ points each one. Afterwards, the mean value of the periodogram $\hat{P}_{x x}(\omega)$, that is a estimator of the power spectrum $P_{x x}(\omega)$ is stated as follows: 


$$
\hat{P}_{x x}(\omega)=\frac{1}{L} \sum_{l=0}^{L-1}\left\{\frac{1}{N}\left|\sum_{n=0}^{N-1} x[n+l N] e^{-j \omega n}\right|^{2}\right\},
$$

where $\omega$ is the normalized discrete frequency. In practice, it is common to use windows to work on smoother functions, so (2) becomes

$$
\hat{P}_{x x}(\omega)=\frac{1}{L} \sum_{l=0}^{L-1}\left\{\frac{1}{N}\left|\sum_{n=0}^{N-1} w[n] x[n+l N] e^{-j \omega n}\right|^{2}\right\},
$$

where $w[n]$ represents the window.

The final form of the mean periodogram does not only depend on the form of the window, but also on $N$ and $L$. Generally, increasing $N$ implies a higher resolution in frequency but sharper and more random spectrum since we have less segments $L$ to calculate the averaging.

When we use a window to calculate the fourier transform of a signal, we give less weight to signal points near the frontiers of the segment. Therefore, we do not exploit as much as possible the entire data epoch. The Welch method improves (3) by overlapping the segments to calculate the mean value. With a $50 \%$ of overlapping e.g., we have:

$$
\hat{P}_{x x}(\omega)=\frac{1}{L} \sum_{l=0}^{L-1}\left\{\frac{1}{N}\left|\sum_{n=0}^{N-1} w[n] x[n+0,5 l N] e^{-j \omega n}\right|^{2}\right\} .
$$

\section{B. The coherence function}

The coherence function between two wide-sense stationary random processes $\mathrm{x}$ and $\mathrm{y}$ is equal to the cross power spectrum divided by the square root of the product of the two auto power spectra. This function can be imaginary and complex. Coherence is formally defined by

$$
\gamma_{x y}(f)=\frac{P_{x y}(f)}{\sqrt{P_{x x}(f) P_{y y}(f)}}
$$

where $f$ is the physical frequency in $\mathrm{Hz}$ and $P_{x y}$, the complex cross power spectrum according to

$$
P_{x y}(f)=\sum_{-\infty}^{+\infty} R_{x y}(m) e^{j 2 \pi f m} .
$$

$P_{x y}(f)$, in addition, is the Fourier transform of the cross correlation function

$$
R_{x y}(\tau)=E[x(n) y(n+\tau)] .
$$

The $x$ and $y$ (EMG signals) are real and $E[]$ denotes the mathematical expectation.

We can also estimate $P_{x y}(f)$ by

$$
\hat{P}_{x y}(f)=\hat{P}_{x x}(f) \cdot \overline{\hat{P}_{y y}}(f)
$$

that is the product of the estimated power spectrum of the first signal by the conjugated of the estimated power spectrum of the second one. $\hat{P}_{x y}(f)$ is a complex function, unlike the estimation of the power spectrum of the signals.

The coherence is a normalized cross-spectral density function so that the magnitude-squared coherence (MSC) is defined by

$$
C_{x y}(f)=\left|\gamma_{x y}(f)\right|^{2}
$$

which lies in the range

$$
0 \leq C_{x y}(f) \leq 1
$$

for all frequencies.

Using (4) and (8), an estimation of $C_{x y}(f), \hat{C}_{x y}(f)$ can be obtained as

$$
\hat{C}_{x y}(f)=\frac{\left|\hat{P}_{x y}(f)\right|^{2}}{\hat{P}_{x x}(f) \hat{P}_{y y}(f)} .
$$

\section{PReliminary Results}

For the first trials we use the first prototype of the TCA system [7] which consists in a portable wireless electronic device, see figure 1.

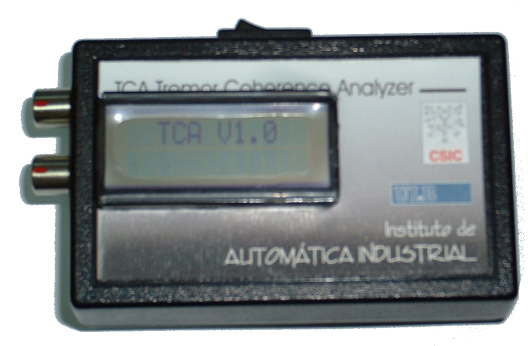

Fig. 1. The TCA portable device used in the experimental trials.

The system has two components. The first component is the portable unit. The portable unit is located on the upper limb and it collects the data from EMG sensors. The surface EMG sensors are located over the flexor and/or extensor muscles. The unit processes the acquired data and shows relevant information in a LCD display. It can also transmit the data to the base Unit, the second component.

The Base Unit is a software platform that analyzes and presents the data to the clinician. The software platform can plot and store received data for a subsequent analysis, which is the scenario for this first trials. It also has a configuration tool to set up the sampling frequency of the acquisition system and the ADC channels to be used. These configuration parameters are transmitted to the portable unit. The system uses Bluetooth technology to communicate the different units.

The system developed in TCA project is still subject to clinical validation. However, preliminary tests with 4 patients have been carried out. These patients suffer from different types of tremor, such as parkinsonian, essential or orthostatic 


\begin{tabular}{|c|c|c|c|}
\hline Patient & Gender & Diagnosis & Tremor $(\mathrm{Hz})$ \\
\hline P1 & M & PD $^{1}$ & $4-6$ \\
\hline P2 & F & Orthostatic & $\sim 15$ \\
\hline P3 & M & PD $^{1}$ and ET & Mixed \\
\hline P4 & F & Diskynesia & Mixed, 3-4 \\
\hline
\end{tabular}

1 Parkinson Desease

2 Essential Tremor

TABLE I

PATIENTS WHO COLLABORATED WITH THE FIRST TRIALS WITH THE TCA SYSTEM.

tremor. In addition, one of the patient presents dyskinesia. The data acquired with the prototype system have been analyzed.

Each patient realized certain number of tasks, usually used in diagnosis of tremor such as finger to finger test, finger to nose, open-close hands among others. A detailed and comprehensive analysis for the entire set of patients and trials is out of the scope of this article. However, in the following paragraphs we will highlight some interesting findings that justify the use of the coherence to study the pathological tremor.

In table IIIwe have the result obtained with patient 3. The fist column shows the main tremor components found in channel 1 , and the same for the second column. The third one indicates the duration in seconds of the task and the fourth one the type of activity. The last columns indicates the frequency whether we have the main coherence values. At last we have to indicate that channel 1 (SEMG sensor) was located on Carpis Radialis Extersor muscle and channel 2 on Triceps muscle.

During tasks 8, 9 and 10 we can see a $11 \mathrm{~Hz}$ oscillator that suddenly appears in channel 1 . This component remains active until task 11, a tapping task, when it disappears. This type of behavior has been found also in P4, becoming a interesting future research topic. The influence of muscle fatigue, stress, drugs and task realization are some of the factors that have to be studied.

- Regarding the signal acquired from patients with parkinson desease, the coherence values increased in muscles of the same limb, with mental tasks during the task realization and the stress produced by the them as Hurtado has reported is his works [5].

- "When both cranial muscles and limb muscles show high coherence values, a supra-spinal mechanism can be suspected". Although the TCA project does not use EEG signals, the first results the sudden presence of oscillators during some specific tasks. This behavior has been found in P3 y P4. The result is a motivation, and presents the coherence as a tool to study the tremor genesis.

- Compensatory strategies is another field that can be studied. The tremor component of $7 \mathrm{~Hz}$ disappeared e.g. during finger to finger task in P4. This type of applications of the coherence represents a goal in the study of the neuromotor system.

- The coherence as a tool will help industry to evaluate

\begin{tabular}{|c|c|c|c|c|c|c|c|}
\hline $\begin{array}{l}\text { CH1 } \\
(\mathrm{Hz})\end{array}$ & $\begin{array}{l}\text { CH2 } \\
(\mathrm{Hz})\end{array}$ & $\mathbf{t}(\mathbf{s})$ & Task & $\mathbf{f}(\mathbf{H z})$ & $\operatorname{Cxy}(f)$ & $\mathbf{f}(\mathbf{H z})$ & $\operatorname{Cxy}(f)$ \\
\hline 8 & 8 & 57.37 & A & 5 & 0.4 & 9 & 0.4 \\
\hline $\begin{array}{l}2.8, \quad 3 \\
\text { and } 5.8\end{array}$ & $\begin{array}{l}6 \text { and } \\
8.2\end{array}$ & 29.7 & B & 5 & 0.8 & 14 & 0.7 \\
\hline 8.5 & 8 & 34.24 & $\mathrm{C}$ & 9 & 0.3 & & \\
\hline 10 & $\begin{array}{l}5.8,6.2 \\
\text { and } 11\end{array}$ & 39 & $\mathrm{D}$ & 9 & 0.3 & 15 & 0.6 \\
\hline 6 & 6 & 55 & E & 6 & 0.55 & 11 & 0.55 \\
\hline 6 & 6 & 36.8 & $\mathrm{~F}$ & 6 & 0.7 & & \\
\hline 6 & 6 & 43.18 & $\mathrm{~F}$ & 6 & 0.9 & 11 & 0.55 \\
\hline $\begin{array}{ll}6 & \text { and } \\
11 & \\
\end{array}$ & 6 & 17.62 & G & 6 & 0.9 & & \\
\hline $\begin{array}{ll}6 & \text { and } \\
11 & \end{array}$ & 6 & 54.74 & $\mathrm{E}$ & 6 & 0.65 & 10.5 & 0.8 \\
\hline $\begin{array}{ll}6 & \text { and } \\
11 & \end{array}$ & 6 & 98.36 & $\mathrm{H}$ & 6 & 0.8 & 10.5 & 0.5 \\
\hline 4 and 6 & 4 and 6 & 52 & I & 6 & 0.9 & & \\
\hline 4 and 6 & 4 and 6 & 39.52 & $\mathrm{I}$ & 6 & 0.9 & & \\
\hline 4 and 6 & 4 and 6 & 35 & $\mathrm{I}$ & 6 & 0.9 & & \\
\hline 6 & 6 & 17 & $\mathrm{G}$ & 6 & 0.9 & 8 & 0.9 \\
\hline
\end{tabular}

Tasks Codes:

A: open-close right hand

B: open-close left hand

$\mathrm{C}$ : prono-supination

D: extended arms + mental task

E: finger to finger

F: finger to nose

G: extended arm

$\mathrm{H}$ : forearm vertical position

I: tapping

TABLE II

RESULTS OBTAINED WITH PATIENT 3 DURING TASKS REALIZATION.

drugs for tremor cancellation among others neuromotor diseases.

\section{CONCLUSIONS}

In this article we have shown preliminary results with the TCA system. We presented one application of the system consisting in sudden oscillators detection. Once the oscillators have been detected we can evaluate coupling between multiple oscillators. Objectively, the coherence itself, can not represent a proof of the presence of a single oscillator that affects multiple muscles but it represents the first trace to these coupling behaviors in tremor genesis.

Regarding the first trials with patients, we have shown the value of the TCA system, a portable tool to asses instantaneously coherence between SEMG signals, to study neuromotor disorders such tremor and oscillators behaviors. The system have many applications an its best goal is the automation of data analysis in real time.

\section{FUTURE WORK}

Next steps in the TCA project include the validation of the signals analysis tools and the study of other types of sensors for physiological monitoring. Validation with patients is also scheduled in the project as a next step. After the first results, tasks and patients scheduling will take into account because of the large amount of neuromotor behaviors that can be studied with the tool. 

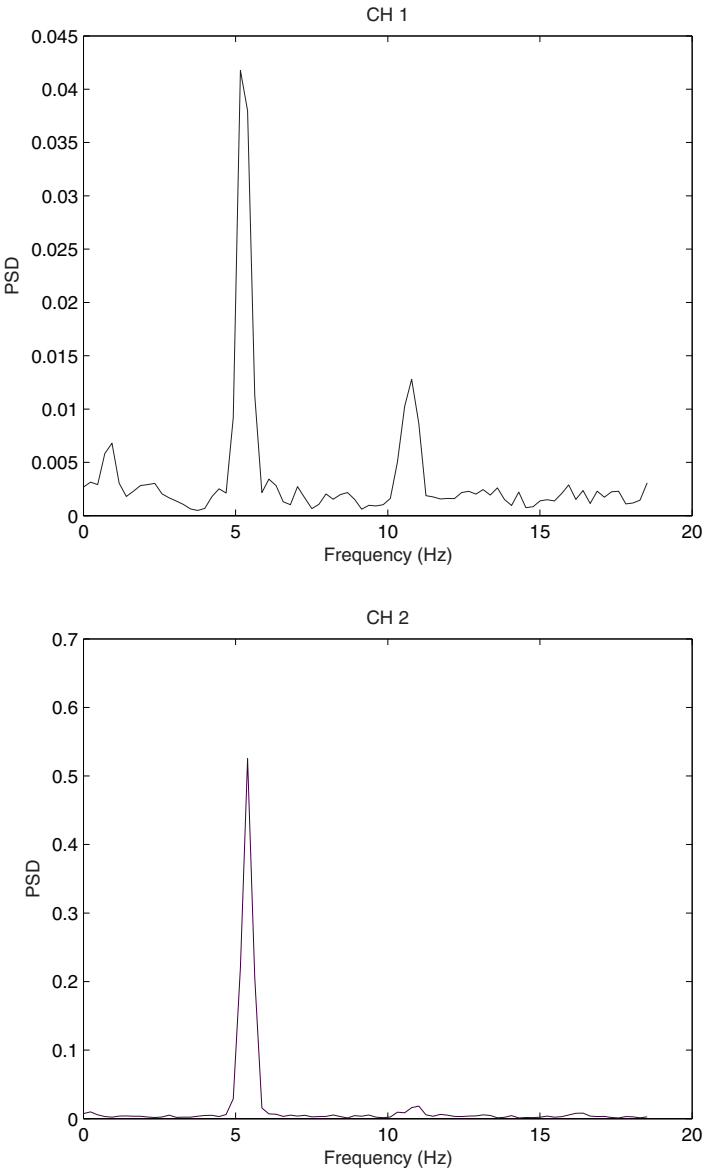

Fig. 2. Power spectral density of channels 1 and 2 corresponding to task 8 of patient 3 . The sudden oscillator of $11 \mathrm{~Hz}$ can be seen in channel 1 .

\section{REFERENCES}

[1] L. Timmermann, J. Gross, M. Dirks, J. Volkmann, H. Freund, and A. Schnitzler, "The cerebral oscillatory network of parkinsonian resting tremor," Brain, vol. 126, 2003.

[2] A.M Amjad, D.M. Halliday, J.R. Rosenberg, and B.A. Conway, "An extended difference of coherence test for comparing and combining several independent coherence estimates: theory and application to the study of motor units and physiological tremor," Journal of Neuroscience Methods, vol. 73, pp. 69-79, 1997.

[3] J. O'Sullivan, J. Rothwell, A. Lees, and P. Brown, "Bilaterally coherent tremor resembling enhanced physiological tremor: report of three cases," Movement Disorders, vol. 17, no. 2, 2002.

[4] S. Kelly, P. Dockree, R.B. Reilly, and I. Robertson, "Eeg alpha power and coherence time courses in a sustained attention task," in Proceedings of the IEEE International Coference on Neural Engineering, March, 2003.

[5] J.M. Hurtado, J.P. Lachaux, and D.J. Beckley, "Inter- and intralimb oscillator coupling in parkinsonian tremor," Movement Disorders, vol. 15, no. 4, pp. 683-691, 2000.

[6] D.M. Halliday, B.A. Conway, S. Farmer, U. Shahani, A. Russell, and J. Rosenberg, "Coherence between low frequency activation of the motor cortex and tremor in patients with essential tremor," The Lancet, vol. $335,2000$.

[7] F. Brunetti, E. Rocon, J.L. Pons, and M. Manto, "The tremor coherence analyzer(tca): A portable tool to assess instantaneous intermuscle coupling in tremor," in 26th International Conference of the IEEE/Engineering in Medicine and Biology Society, 2004. 